\title{
Disturbed Interplay Between Mid- and High-Level Vision in ASD? Evidence from a Contour Identification Task with Everyday Objects
}

\author{
Kris Evers $\cdot$ Sven Panis $\cdot$ Katrien Torfs $\cdot$ \\ Jean Steyaert $\cdot$ Ilse Noens $\cdot$ Johan Wagemans
}

(C) Springer Science+Business Media New York 2013

\begin{abstract}
Atypical visual processing in children with autism spectrum disorder (ASD) does not seem to reside in an isolated processing component, such as global or local processing. We therefore developed a paradigm that requires the interaction between different processes-an identification task with Gaborized object outlines-and applied this to two age groups of 6-to-10 and 10-to-14 year old children with and without ASD. Event history analyses demonstrated an identification disadvantage in the ASD group, which remained quite stable during the temporal unfolding of the outline. The typically developing group particularly
\end{abstract}

Electronic supplementary material The online version of this article (doi:10.1007/s10803-013-1931-7) contains supplementary material, which is available to authorized users.

K. Evers $(\bowtie) \cdot$ S. Panis $\cdot$ K. Torfs · J. Wagemans

Laboratory of Experimental Psychology, KU Leuven,

Tiensestraat 102, box 3711, 3000 Leuven, Belgium

e-mail: kris.evers@psy.kuleuven.be

K. Evers · J. Steyaert

Department of Child Psychiatry, UPC-KU Leuven, Leuven,

Belgium

K. Evers · J. Steyaert · I. Noens · J. Wagemans

Leuven Autism Research (LAuRes), KU Leuven, Leuven,

Belgium

J. Steyaert

Department of Clinical Genetics, University Hospital Maastricht,

Maastricht, The Netherlands

I. Noens

Parenting and Special Education Research Unit, KU Leuven, Leuven, Belgium

I. Noens

Psychiatric and Neurodevelopmental Genetics Unit,

Massachusetts General Hospital, Boston, MA, USA outperformed the ASD group when more complex contours were shown. Together, our results suggest that the interplay between local and global processes and between bottom-up and top-down processes is disturbed in ASD.

Keywords Autism spectrum disorder (ASD) . Visual perception - Object identification - Weak central coherence theory - Enhanced perceptual functioning hypothesis - Bottom-up versus top-down . Gabor $\cdot$ Local versus global processing

\section{Introduction}

Individuals with an autism spectrum disorder (ASD) are characterized by qualitative impairments in social reciprocity, communication and repetitive and stereotyped behavioral patterns (DSM-IV-TR; American Psychiatric Association 2000). In 1943 already, Kanner associated ASD with a local perceptual style, by inferring that children with "autistic aloneness" have an "inability to experience wholes without full attention to the constituent parts". Current DSM-IV-TR criteria mention "persistent preoccupation with parts of objects" as one of the manifestations of "restricted, repetitive, and stereotyped patterns of behaviors, interests, and activities", and sensory symptoms obtain a more prominent place in the new version of the DSM (DSM-5; Wing et al. 2011). Thus, although not central in the official DSM criteria, perceptual symptoms were often described in children with ASD and received considerable attention in theoretical accounts and recent empirical work.

Atypical perceptual patterns have been the focus of two prominent (neuro)cognitive theories in ASD, namely the weak central coherence (WCC) theory (Frith and Happé 
1994; Happé and Booth 2008; Happé and Frith 2006) and the enhanced perceptual functioning (EPF) hypothesis (Mottron and Burack 2001; Mottron et al. 2006). Both WCC and EPF attribute a more locally than globally oriented processing style to individuals with ASD, consistent with results of several studies (for reviews, see Behrmann et al. 2006; Dakin and Frith 2005; Simmons et al. 2009). WCC originates in the observation of a rather detailedfocused cognitive style in individuals with ASD, combined with deficits in automatic Gestalt formation ('not seeing the forest through the trees'). Unlike WCC, EPF focuses on the enhanced low-level perception of people with ASD, without making any further claims about global processing. Both theories are still further refined in confrontation with recent empirical literature, and thus, differences between them decreased since their first descriptions. Moreover, both the role and influence of this atypical perception on other domains of functioning in ASD, are still under debate (for a critical discussion about the boundaries of the explanatory power of WCC, see Ronald and Happé 2008, and also Happé and Booth 2008, and for the importance of an atypical, veridical, perceptual style in savantism, see Mottron et al. 2013).

The empirical work on local and global processing in ASD started with a classical study by Shah and Frith (1983), who showed that children with ASD detected a target shape embedded in a larger figure better than controls (so-called Embedded Figures Test). Later studies confirmed the atypical visual processing style using visual illusions, block design tasks, visual search tasks, hierarchical figures, copy tasks, and discrimination tasks (for reviews, see Behrmann et al. 2006; Dakin and Frith 2005; Simmons et al. 2009). However, not all studies confirmed a deficit in global processing style, nor superior detail processing. Results seem to depend on participant characteristics (e.g., age, intelligence level), task demands (e.g., focus on a global or local processing level, implicit or explicit instructions: e.g., Evers et al. 2011), and stimulus characteristics (e.g., basic, low-level visual stimuli such as contrast or luminance detection versus more complex, semantically meaningful stimuli). Moreover, the results are sometimes difficult to interpret due to the vague conceptualization of local and global visual processing and their many different operationalizations in specific experimental variables (for recent empirical work on concept validation of local/global measures, see Dale and Arnell 2013; Milne and Szczerbinski 2009).

Neurocognitive theories, such as WCC and EPF, emphasized either reduced top-down influences (Happé and Booth 2008; Loth et al. 2010; Mitchell et al. 2010), or increased bottom-up processes in ASD (Mottron et al. 2006). In line with this point of departure, the literature has mainly been searching for tasks on which children with
ASD show deficits in global processing and for tasks on which they outperform typically developing (TD) children (by using a superior local strategy). However, this search has been complicated by the factors mentioned above. Moreover, there are indications in the literature that the differences in visual processing in children with ASD do not reside in a specific isolated processing component. Indeed, previous research focusing on specific component processes of perceptual grouping mostly did not show differences between children with and without ASD (i.e., no grouping disadvantages using low-level stimuli, e.g., Blake et al. 2003; Del Viva et al. 2006; Kemner et al. 2007). Perhaps the atypicality of visual processing in ASD resides more in the interaction between top-down and bottom-up (stimulus-driven) mechanisms, which is less developed or less flexibly tailored to the stimuli and tasks at hand in people with ASD (Barttfeld et al. 2011; Samson et al. 2012; Wass 2011).

We have developed a paradigm that requires the interplay between different component processes and may therefore be suitable to test this hypothesis. In a previously developed object identification task, participants had to identify an everyday object from tiny fragments along its external contour, which were gradually shown for a longer time (Panis and Wagemans 2009) or which were gradually expanded until the full contour became visible (Torfs et al. 2010). These studies showed a nice interplay between bottom-up grouping of contour fragments and top-down matching of possible object representations, which could be tracked dynamically over time (i.e., with increasing stimulus duration or during the unfolding of the object outline) and related to well-controlled stimulus factors (e.g., object category, symmetry, and complexity). The present paradigm adds a number of factors by using Gabor elements that could be aligned along the contour (relating to the extensive literature on contour linking using the "snake detection" paradigm; for reviews, see Hess and Field 1999; Hess et al. 2003) or that could have the same orientation within the surface area bounded by the contour (relating to the extensive literature on perceptual grouping and figure-ground organization; for a recent review, see Wagemans et al. 2012). Moreover, by embedding these target elements belonging to the object to be identified in a completely irregular background of random Gabor elements, the present paradigm introduces the kind of clutter that necessitates segregation processes in natural images. Finally, the display is changing dynamically over time, with the alignment of the Gabor elements along the contour and inside the surface gradually increasing, again leading to some kind of "unfolding of the stimulus" in the sense of a gradual increase of clarity of the contour grouping, texture segregation, and object identity (see Fig. 1 for an example and http://www.gestaltrevision.be/en/resources/ 
supplementary-material for an animated version with the same presentation speed as used in the actual experiment).

There is a rich set of processes involved in trying to identify an object from such a dynamic series of Gabor displays with an embedded contour and surface. By binding disjoint local elements on the basis of their proximity, similarity, and so forth, participants can group the local Gabor elements into a contour and a surface. These early grouping processes help them to delineate a possible object shape and segregate that from the background, which may then lead to the construction of an object representation, which in turn is matched to memory, resulting in object identification. Then, a word label can be assigned to this object representation, which leads to object naming. Note that these processes do not have to operate in a strict sequential fashion (Humphreys et al. 1988). Indeed, it is quite likely that there is an intricate interplay between these bottom-up and top-down processes. For instance, there will be interactions between contour and surface grouping, which will together interact with figure-ground segregation and the formation of an object representation, and with semantic processes (Sassi et al. 2010). Feedback from candidate object representations can facilitate or hamper grouping and segregation and so forth (Panis and Wagemans 2009). In difficult or ambiguous cases, several iterations within this cycle of feedforward and feedback processing can be necessary, in which different object representations may be constructed and tested against the available evidence.

By experimentally slowing down the contour build-up to $20 \mathrm{~s}$ (from fully random to maximum alignment in 20 frames of $1 \mathrm{~s}$ each) we can investigate the temporal dynamics of these processes and their interplay. By using event history analysis (Allison 2010; Panis and Hermens 2013; Panis and Wagemans 2009; Singer and Willett 2003) to analyze the shape of the response time distributions, we can study the temporal dynamics of the identification

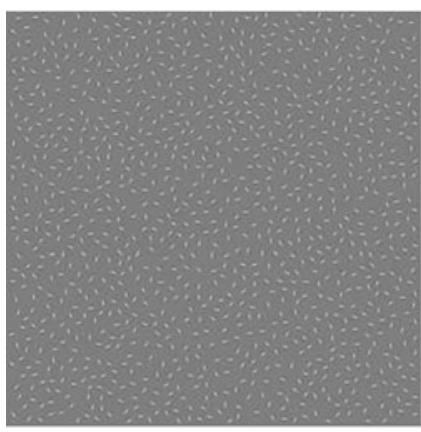

Frame 1

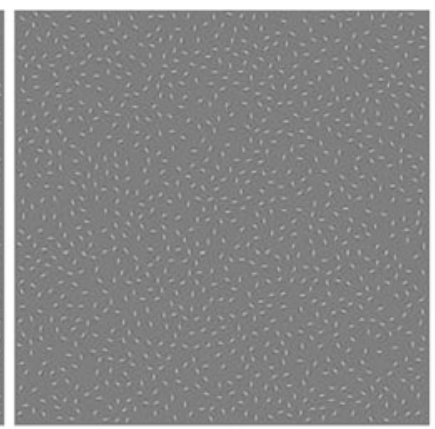

Frame 6
Fig. 1 Examples of frames within the build-up. In total, 20 frames were used for the transition from completely random Gabor pattern towards a fully organized version. Here a selection is presented, process and its component processes over time. Moreover, it allows us to evaluate the influence of certain predictors (e.g., stimulus properties such as complexity, object category, symmetry, and also participant group) on the shape of the response time distribution during the stimulus build-up sequence. Our stimuli consisted of contours of 56 objects, representative for the type of objects and shapes one encounters in daily life. At the same time, this heterogeneous set of contours is well-controlled on several low(e.g., number of elements in the background and on the contour surface, contour length), mid- (e.g., compactness, and homogeneity), and high-level stimulus characteristics (e.g., identifiability). Previous research in TD adults already revealed the impact of stimulus properties on object identification (e.g., Panis and Wagemans 2009; Torfs et al. 2010). For instance, fragmented outlines of shapes with a low visual complexity are known to have an early, bottom-up grouping advantage (in comparison to complex outlines). However, once these simple shape outlines are grouped, they will activate more object representations (because they have a higher a priori occurrence), resulting in a slower top-down matching process (Donderi 2006; Gerlach et al. 2004, 2006; Panis and Wagemans 2009). Therefore, manipulating mid-level (e.g., complexity, symmetry) and high-level (e.g., object category: manmade versus natural) stimulus properties may provide us with a more detailed picture of the influence of these properties on the identification process in both participant groups. Not much research is focusing on the influence of these specific mid- or high-level factors in ASD. Yet, two contradicting studies are demonstrating the atypical impact of mirror symmetry on performance in individuals with ASD, by demonstrating a lower (Perreault et al. 2011) or higher (Falter and Bailey 2012) detection threshold for symmetry in children with ASD.

In sum, we believe this novel paradigm has the potential to reveal possible differences in the interplay between

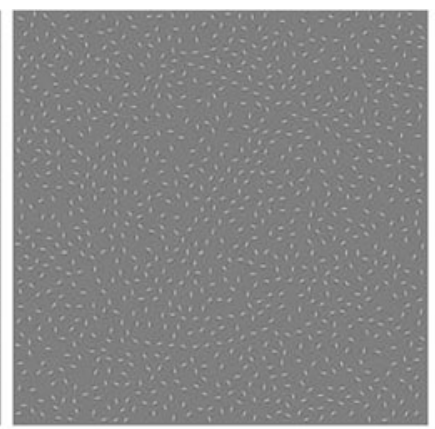

Frame 11

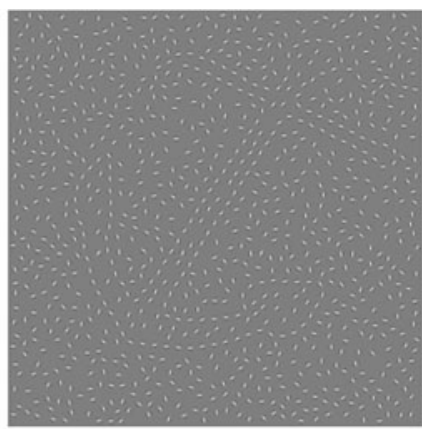

Frame 16

ranging from completely unorganized Gabor pattern (frame 1) towards a more organized version (frame 16) 
bottom-up and top-down processes while participants carry out a task in which they are trying to make sense of a changing input with variable levels of noise and structure. If our hypothesis is correct and a major difference between visual processing in individuals with and without ASD resides in the interplay between local and global processes and between bottom-up and top-down processes, then this paradigm should be able to reveal a difference between the two groups. In addition, the event history analysis would show how this possible difference is affected by any of the stimulus characteristics under experimental control, and how this changes over time.

\section{Methods}

Although Experiment 1 and Experiment 2 have a similar general set-up, both experiments differed in two respects: the participants' age (10-to-14-year olds in Experiment 1, and 6-to-10-year olds in Experiment 2), and the details of the stimulus build-up procedure (see "Stimuli"). Both experiments were conducted sequentially, in two different data collection waves, allowing us to use the results of Experiment 1 to make small adaptations (such as the stimulus build-up) in Experiment 2. The use of two age groups is interesting to test for the robustness and generality of the effects, and the two samples were chosen to capture a large developmental window at school age.

\section{Participants}

Two groups of children participated in this study. The ASD group was formally diagnosed based on the DSM-IV-TR criteria (American Psychiatric Association 2000) by a multidisciplinary team or a child psychiatrist. These children were recruited via the Autism Expertise Centre at the University Hospital and via a special needs school for children with ASD. Diagnoses were confirmed with the Autism Diagnostic Observation Scale (ADOS; Gotham et al. 2008; Lord et al. 2000) in 41 out of 44 cases (matched samples: Experiments 1 and 2: 19/21 and 21/23 ASD participants, resp.). ADOS severity scores, which are calibrated scores ranging between 1 and 10 (Gotham et al. 2009), are included in the demographics table (Table 1). Severity scores 4 and 5 are considered representative of an ASD classification. The TD group was representative for the general population and recruited through mainstream schools, personal contacts and advertisements. According to verbal reports from the parents, these children had no child psychiatric disorder and no first-degree family member with ASD. In addition, the average raw score on the Dutch Social Responsiveness Scale (SRS; Constantino 2002; Roeyers et al. 2012) in the TD group was 24
$(\mathrm{SD}=13.76$, Experiment 1$)$ and $24.94(\mathrm{SD}=10.22$, Experiment 2), resp., both far below T70 (i.e., a norm score of $2 \mathrm{SD}$ above the mean, corresponding to a raw total score of 60 for boys and of 55 for girls).

We only included 10-to-14-year old boys (Experiment 1; detailed characteristics in Table 1) and 6-to-10-year old boys and girls (Experiment 2; detailed characteristics in Table 1) with an IQ $\geq 70$ (VIQ, PIQ and TIQ). Intelligence was assessed with an abbreviated version (Sattler 2001) of the Wechsler Intelligence Scale for Children (WISC-III-R; Wechsler 1992), comprising the following subtests: Block Design, Similarities, Picture Completion and Vocabulary. In total, 49 children with ASD (Experiment 1: $\mathrm{n}=26$, and Experiment 2: $\mathrm{n}=23$ ) and $60 \mathrm{TD}$ children (Experiment 1: $\mathrm{n}=24$, and Experiment 2: $\mathrm{n}=36$ ) participated. We selected a subsample of children with ASD and TD children, to create two group-wise matched participant groups matched based upon age and intelligence. None of these children used neuroleptics (as reported by one of their parents). All children reported normal or corrected-to-normal vision and were Dutch-speaking.

\section{Stimuli}

The stimulus set is a modified version of the standardized set of 260 line drawings of everyday objects by Snodgrass and Vanderwart (1980). Outlines were derived from the object silhouettes, which were created by filling-in the interior surface of the original line drawings (for further technical details, see Wagemans et al. 2008).

In the present study, the object outlines were embedded within arrays of non-overlapping Gabor elements on a uniform grey background. A Gabor element is defined as the product of a circular Gaussian (standard deviation of $.08 \mathrm{deg}$ ) and a sine wave luminance grating frequency of 3.57 cycles/deg. Along each object outline, Gabor patches were oriented on equidistant locations. Object exterior was filled with randomly oriented Gabor patches. The orientation of the Gabor patches on the object interior was parallel to the main axis orientation (for further technical details, see Sassi et al. 2010). The orientation of the Gabor elements changed with time, resulting in a transition from fully random (only Gabor elements with random orientation) to fully organized (random exterior, organized interior and outline) in 20 steps, each presented $1 \mathrm{~s}$ per frame. This transition was organized linearly in Experiment 1 and nonlinearly in Experiment 2, with faster transitions early in the sequence and slowed down transitions later on, so that subsequent steps towards emerging organizations were smaller in the second half of the sequence. Further details of the build-up of these stimuli and examples of the stimuli can be found in Fig. 2 and in Appendix 1. In Fig. 1 (above) a static example of the stimulus build-up is displayed (see 
Table 1 Descriptive statistics for age (in months), performance, verbal and total IQ scores, SRS total scores and ADOS severity scores of the ASD and TD group (Experiments 1 and 2)

\begin{tabular}{|c|c|c|c|c|c|c|}
\hline & Age & PIQ & TIQ & VIQ & SRS & ADOS \\
\hline \multicolumn{7}{|l|}{ Experiment 1} \\
\hline \multicolumn{7}{|l|}{ Full group } \\
\hline \multicolumn{7}{|c|}{ ASD group $(n=26)$, only boys } \\
\hline Mean & 151.73 & 96.77 & 98.79 & 100.81 & 79.67 & 5.25 \\
\hline SD & 16.40 & 14.59 & 14.43 & 20.15 & 24.09 & 1.59 \\
\hline Range & $127-206$ & $71-134$ & $72-132$ & $70-146$ & $41-133$ & $3-10$ \\
\hline \multicolumn{7}{|c|}{ TD group $(n=24)$, only boys } \\
\hline Mean & 151.54 & 110.00 & 111.21 & 112.42 & 24.00 & N/A \\
\hline SD & 14.09 & 13.76 & 10.08 & 11.81 & 13.76 & N/A \\
\hline Range & $132-179$ & $83-132$ & $91.5-130.5$ & $97-135$ & $7-56$ & N/A \\
\hline$p$ value (2-sided $t$ test) & .4686 & .7828 & .0883 & .0165 & $<.0001$ & N/A \\
\hline \multicolumn{7}{|l|}{ Matched group } \\
\hline \multicolumn{7}{|c|}{ ASD group $(n=21)$, only boys } \\
\hline Mean & 152.76 & 101.38 & 102.93 & 104.48 & 79.94 & 5.11 \\
\hline SD & 16.24 & 11.72 & 12.44 & 19.35 & 25.05 & 1.66 \\
\hline Range & $128-206$ & $80-134$ & $79.5-132$ & $70-146$ & $41-133$ & $3-10$ \\
\hline \multicolumn{7}{|c|}{ TD group $(n=20)$, only boys } \\
\hline Mean & 152.15 & 106.95 & 108.63 & 110.30 & 22.84 & N/A \\
\hline SD & 14.39 & 12.68 & 8.61 & 11.38 & 11.96 & N/A \\
\hline Range & $132-179$ & $83-129$ & $91.5-126.5$ & $97-135$ & $7-43$ & N/A \\
\hline$p$ value (2-sided $t$ test) & .8993 & .1519 & .0978 & .2502 & $<.0001$ & N/A \\
\hline
\end{tabular}

Experiment 2

Full group

ASD group ( $\mathrm{n}=23), 20$ boys:3 girls

\begin{tabular}{|c|c|c|c|c|c|c|}
\hline Mean & 103.87 & 103.13 & 102.76 & 102.39 & 94.64 & 5.39 \\
\hline SD & 13.19 & 14.69 & 11.77 & 13.18 & 21.16 & 1.78 \\
\hline Range & $83-123$ & $77-134$ & $84.5-130.5$ & $76-135$ & $44-135$ & $2-9$ \\
\hline \multicolumn{7}{|c|}{ TD group $(\mathrm{n}=36), 27$ boys:9 girls } \\
\hline Mean & 102.17 & 106.72 & 109.81 & 112.89 & 24.94 & N/A \\
\hline SD & 14.46 & 11.87 & 9.19 & 11.38 & 10.22 & N/A \\
\hline Range & $80-125$ & $83-134$ & $90-128$ & $89-135$ & $8-56$ & N/A \\
\hline$p$ value (2-sided $t$ test) & .6499 & .3062 & .0128 & .0019 & $<.0001$ & N/A \\
\hline
\end{tabular}

Matched group

ASD group $(\mathrm{n}=23) 20$ boys:3 girls

\begin{tabular}{|c|c|c|c|c|c|c|}
\hline Mean & 103.87 & 103.13 & 102.76 & 102.39 & 94.64 & 5.39 \\
\hline SD & 13.19 & 14.69 & 11.77 & 13.18 & 21.16 & 1.78 \\
\hline Range & $83-123$ & $77-134$ & $84.5-130.5$ & $76-135$ & $44-135$ & $2-9$ \\
\hline \multicolumn{7}{|c|}{ TD group $(\mathrm{n}=25), 22$ boys:3 girls } \\
\hline Mean & 100.92 & 104.68 & 106.26 & 107.84 & 25.33 & N/A \\
\hline SD & 14.70 & 12.16 & 8.32 & 9.16 & 10.60 & N/A \\
\hline Range & $80-125$ & $83-132$ & $90-119.5$ & $89-122$ & $13-56$ & N/A \\
\hline$p$ value (2-sided $t$ test) & .4674 & .6915 & .2450 & .1070 & $<.0001$ & N/A \\
\hline
\end{tabular}

In Experiment 1, SRS questionnaires from 5 children were missing or incomplete (full group: ASD: $n=4$, TD: $n=1$; matched group: ASD: $\mathrm{n}=4$; TD: $\mathrm{n}=1$ ). In Experiment 2, SRS questionnaires from 6 children were missing or incomplete (full group: ASD: $\mathrm{n}=1$; TD: $\mathrm{n}=5$; matched group: ASD: $n=1$, TD: $n=4$ ) 


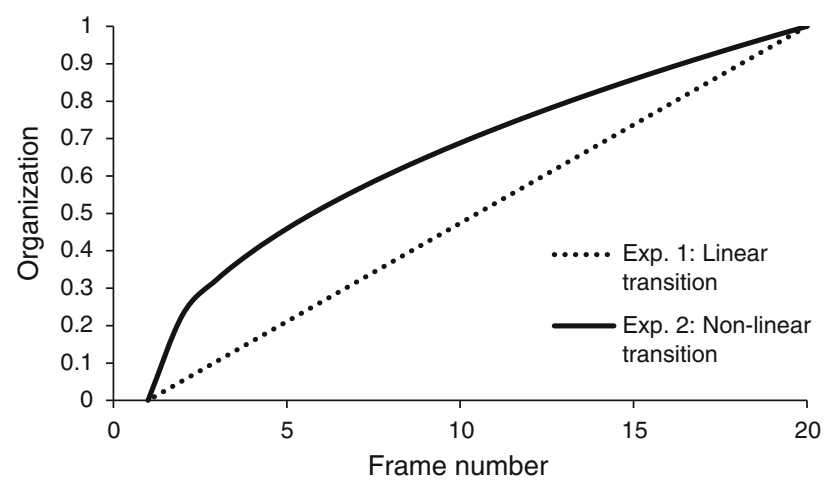

Fig. 2 Comparison of build-up of stimuli, illustrating the relationship between the level of organization of the Gabor patches (vertical axis), which ranges from 0 (fully random) to 1 (fully organized), and the Frame number (horizontal axis), for Experiment 1 (linear transformation; dotted line) and Experiment 2 (non-linear transformation; full line)

http://www.gestaltrevision.be/en/resources/supplementarymaterial for an animated version with the same presentation as used in the actual experiment).

We selected 56 stimuli (see Table 4 in Appendix 2, Supplementary material) with high identification rates in the fully organized version (above $70 \%$ ), as determined in a large normative study by Sassi et al. (2010), comprising 28 outlines of natural objects (including animals, fruits, vegetables and body parts) and 28 outlines of manmade objects (including vehicles, furniture, clothing). The two groups of outlines did not differ in the following stimulus characteristics: mean identification rate, average contour length, average number of elements on the surface, average number of elements in the background, average compactness, and on mean stimulus homogeneity (see Table 5 in Appendix 2, Supplementary material).

\section{Apparatus}

All stimuli were stored in digital form (as bmp files) and presented within the E-prime environment (E-prime version 1.1.41. PST Inc.). Children were seated approximately $57 \mathrm{~cm}$ from a 17-inch computer screen, set to pixel resolution 1,024 by 768 (16-bit color) at $75 \mathrm{~Hz}$ refresh rate. Windows XP was used as operating system. Mouse and keyboard were connected via USB connections to the PC.

\section{Procedure}

With written informed consent of their parents, participants were tested individually in a quiet room. Because of pragmatic reasons, this experiment was incorporated within a larger series of experiments on visual processing and emotion processing. After giving the instructions and only if all questions children might have about them were overcome, the step-by-step-practice protocol began. Participants were instructed to respond (by clicking the mouse) as soon as they could identify the object, even if they were not completely sure about the identification.

All trials started with a $1 \mathrm{~s}$ fixation cross, followed by a frame with fully randomized Gabor elements. Build-up of the Gaborized object outline, frame after frame continued until an answer was provided. Every frame was presented for $1 \mathrm{~s}$. When participants clicked on the mouse, the stimulus disappeared and an answer screen appeared. Participants responded verbally, their answers were entered on the keyboard by the experimenter, and feedback about correctness was given. In case of a correctly answered trial, the build-up was aborted and the experiment proceeded with the next trial. In case of an incorrectly responded trial, build-up of the Gaborized outline proceeded further until another answer was provided, or until the build-up was completed. In that case, participants could give one last answer, and then the next trial began (for an illustration of the procedure, see Fig. 3). The build-up of the experimental trials was identical to that of the practice trials. In total, participants could thus provide answers at 21 time frames (20 frames +1 after build-up).

This experiment consisted of 4 practice trials (same trials for all participants; comprising 2 natural and 2 manmade objects) and 56 experimental trials (28 natural and 28 manmade objects), with a short break after 30 trials. Trial order was individually randomized.

\section{Scoring}

We developed clear scoring rules throughout this study (for more details, see Appendix 2), which were used as criteria to score responses as correct or incorrect, by slightly adapting previously developed scoring rules (Wagemans et al. 2008). Answers were scored manually. Given that different experimenters were engaged in this experiment, all recordings and their scores were checked afterwards. Incorrectly scored items were corrected (in the case of correct items mistakenly scored as incorrect) or deleted (in the case of incorrect items mistakenly scored as correct). For every participant-object combination, all answers, their accuracy and their frame number was recorded.

\section{Event History Analysis}

Considering correct identification as the event of interest, and frame of identification as a discrete time measurement, we can analyze our discrete time-to-event data with discrete time event history analysis (Allison 2010; Singer and Willett 2003). This technique has two major advantages in the context of our study. First, event history analysis provides us with the opportunity to investigate the time-course 
Fig. 3 Illustration of the experiments' procedure. After the $1 \mathrm{~s}$ presented fixation cross, a frame with randomized Gabor elements was presented. The frame-by-frame build-up of the Gaborized object outline (all frames were presented for $1 \mathrm{~s}$ ) continued until an answer was provided. In case of a correctly answered trial, the build-up was aborted and the experiment proceeded with the next trial (full grey arrow). In case of an incorrectly answered trial, build-up continued until another answer was provided or until the build-up was completed

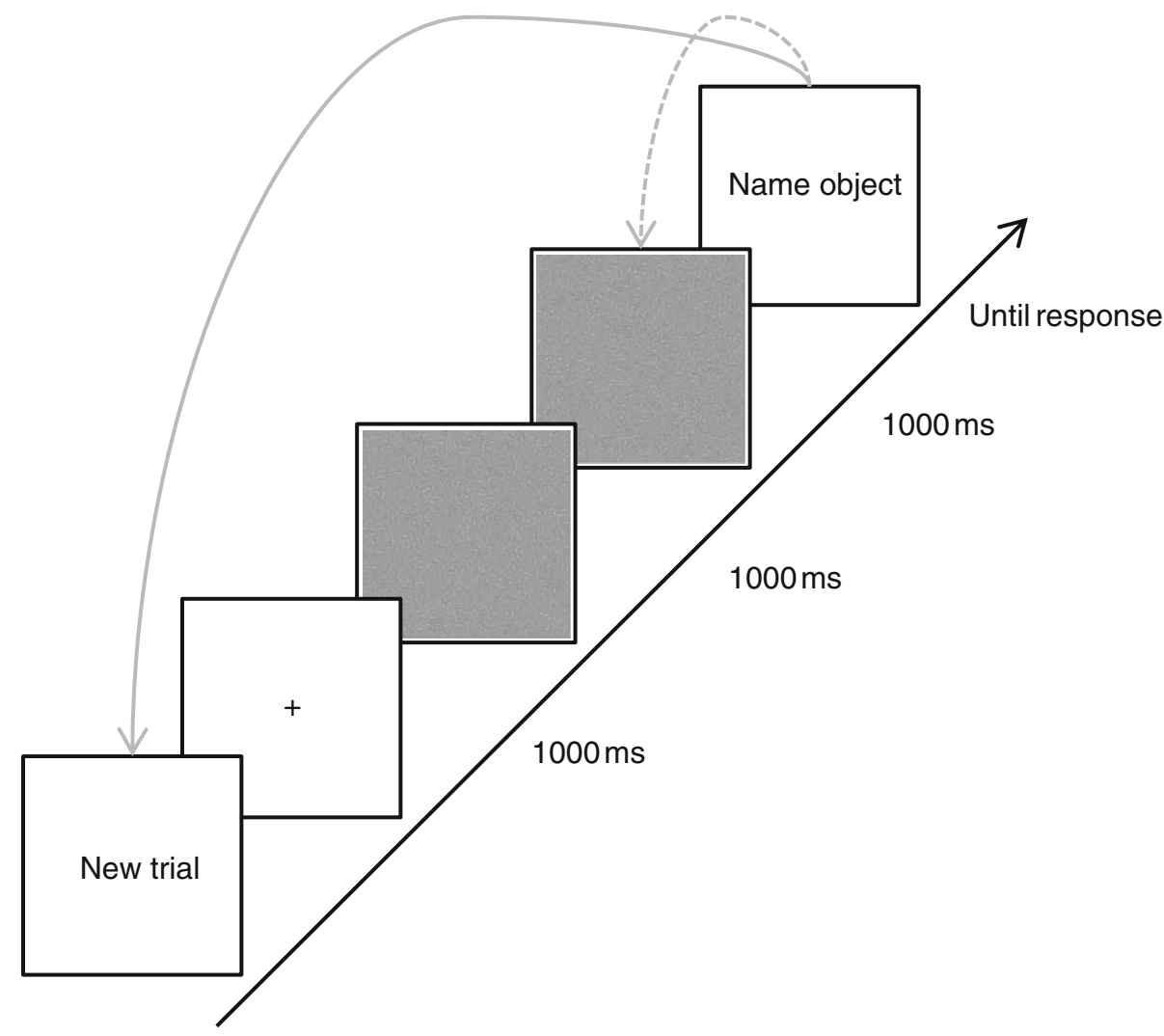

of different predictors (i.e., object characteristics, participant group) by studying their effects on different parts of the reaction time distribution. Since previous research already indicated that different object characteristics have an influence on the time-course of grouping and matching operations, which are at the basis of correct object identification, it seems crucial to investigate the conditional probability of identification throughout time in our ASD sample. Second, in approximately $9 \%$ of all trials (Experiment 1: ASD group: $10.89 \%$, TD group: $7.05 \%$; Experiment 2: ASD group: $10.72 \%$, TD group: $6.86 \%$ ), participants did not correctly identify the object after the last frame, i.e., correct identification (the target event in our study) did not take place during data collection. These data are thus said to be right-censored data (Allison 2010). Whereas most traditional data-analysis techniques simply ignore right-censored observations, event history analysis takes it into account.

We will only briefly describe the main features of event history analysis. For further details, we refer to Singer and Willett (2003), and to Panis and Wagemans (2009). The 56 objects were treated as experimental units, whereas the participants acted as repeated measures of these experimental units. In an event history analysis, the distribution of event occurrence (i.e., correct identification) is summarized by two statistics: the hazard function and the survivor function. The survivor function gives the probability that the object on trial $i$ will 'survive' (i.e., not be correctly identified) during time bin $j$. The discrete time hazard probability $h\left(t_{i j}\right)$ refers to the conditional probability that the object on trial $i$ will be correctly identified in time bin $j$, given that it has not been correctly identified in earlier time frames of trial i. Hazard probability ranges between 0 and 1 . Therefore, we apply a transformation before generalized linear models (GLM) for repeated measurements are fitted to the data. The nonlinear, asymmetric complementary log-log link function was used $(\operatorname{cog} \log$ hazard $=\ln (-\ln (1-$ hazard $)))$, because this transformation is most appropriate when discrete time methods are used to measure a continuous underlying metric of time (Panis and Wagemans 2009; Singer and Willett 2003; Torfs et al. 2010). To fit the hazard model, the surveylogistic procedure of SAS 9.3 was used.

We included the following four dichotomous predictors in our model: participant group ( 1 for the ASD group, and 0 for the TD group), object category ( 1 for natural objects, and 0 for manmade objects), global symmetry ( 1 for symmetrical objects, and 0 for asymmetrical ones), and homogeneity of the object outline (we recoded homogeneity into a dichotomous variable, based upon a median split: 1 for the most homogeneous (simplest) half of the objects, and 0 for the least homogeneous (most complex) ones). 
We used a backward model selection procedure, applying the hierarchical principle, and starting with a full model, which included linear, quadratic and cubic main effects of time, all main effects of the predictors, and all two-way interactions between predictors. To evaluate the variability in time of these effects, we also included the interactions between the linear and quadratic effects of time and the other effects. The earliest frame numbers, in which no identifications were made, were excluded from the analyses (Experiment 1: exclusion frame number 1-10; Experiment 2: exclusion frame number 1-6), and the hazard model was fitted on the frame number with a survival probability of approximately .50 (Experiment 1: frame number 14, Experiment 2: frame number 11). Afterwards, the selected model was also fitted on the other frame numbers.

In the following sections, we will present the final (reduced) hazard model. The first five parameters in the tables (in Appendices 4 and 6) always describe the shape of the baseline cloglog hazard function. This baseline shows the evolvement of the hazard for the baseline condition, i.e., the average TD participant (participant group $=0$ ), with a complex (homogeneity $=0$ ), asymmetrical (symmetry $=0$ ) and manmade (natural $=0$ ) object. Parameter estimates are expressed in cloglog hazard units. After exponentiating the parameter estimates, they can be interpreted as the ratio of the hazard in two groups-the one for which the predictor value is 1 and the one for which it is 0 .

\section{Results}

Although the results of Experiment 1 and 2 will be presented separately (in Experiment 1: 10-to-14-Year Olds and Experiment 2: 6-to-10-Year Olds sections, resp.), both sections have a similar outline. First, some general statistics will be presented (in "Descriptive Statistics and Overall Results" sections): the recognition rate (Do both participant groups differ in the percentage correctly identified contours?) and the number of attempts (Do both participant groups differ in the number of answers they provide?). In addition to these general summary statistics, results from the event history analyses will be presented (in "Event History Analysis: General" and "Event History Analysis: Effect of Participant Group" sections). Instead of evaluating an effect at a certain moment, event history analyses provide us with a detailed picture of the evolution of the recognition rate during the whole time-course of stimulus build-up. In other words: What is the probability that you recognize the contour (given that you did not do so before)? And how does this conditional probability (the socalled hazard probability) evolve over time? Note that time is measured in frame numbers in the context of this experiment. Hence, our question could be reformulated as follows: How does the hazard probability evolve in function of the organization of the elements into an organized contour?

The event history sections begin with specifying our prediction model, and describing the baseline of the hazard probability (beginning of sections "Event History Analysis: General"). This baseline hazard function refers to the conditional probability that the average 'baseline' object (i.e., complex, asymmetrical, manmade object) is recognized by the average 'baseline' participant (i.e., the average TD participant) during a certain time frame, given that it has not been identified yet. In the next sections, we will present the effects of the different predictors (such as participant group, and the stimulus characteristics), the interactions between the predictors, and the evolution of these effects throughout the stimulus build-up.

\section{Experiment 1: 10-to-14-Year Olds}

\section{Descriptive Statistics and Overall Results}

Although the overall correct identification rate tended to be slightly higher in the TD group $(\mathrm{M}=92.95 \%, \mathrm{SD}=.04)$ than in the ASD group $(\mathrm{M}=89.11 \%, \mathrm{SD}=.10)$, the difference was not statistically significant (Mann-Whitney U-test: $\mathrm{z}=1.63, p=.1025)$. There was also no evidence for fewer or more attempts in providing an answer in the ASD group: both groups provided an equal number of attempts per trial (ASD: $\mathrm{M}=1.09, \mathrm{SD}=.08$; $\mathrm{TD}$ : $\mathrm{M}=1.07, \mathrm{SD}=.06 ;$ Mann-Whitney U-test: $\mathrm{z}=-.51$, $p=.6088)$.

\section{Event History Analysis: General}

The final (reduced) hazard model fitted at frame number 14 comprised 20 parameters, displayed in Table 6 (Appendix 3) together with their parameter estimates, standard errors, Chi square values, and $p$ values. In Appendix 4, the model based hazard plots of all conditions are displayed.

The hazard in the baseline condition (described by parameter 1-5, and displayed by the full line in Fig. 4), was rather low for the earlier frames, and slowly increased to .20 around frame number 15 . In other words, the conditional probability that the average TD participant correctly identifies a baseline object (i.e., complex, asymmetrical, manmade) between the 15th and 16th frame is estimated to be about $20 \%$ (given that the object has not been identified correctly before the start of frame 15). This conditional hazard probability increased sharply across the last frames, and reached approximately $31 \%$ at the final frame number. This late increase in the hazard of event occurrence reflects the fact that observers could inspect the 
final $100 \%$ collinear version, thus increasing the chance that the correct name will be given after all. The graph in Fig. 4 also shows that the average ASD participant (dotted line) had a lower conditional hazard probability, i.e., the average ASD participant had a lower probability to correctly identify the object at all frame numbers. (This effect will be substantiated statistically in the next section.)

\section{Event History Analysis: Effect of Participant Group}

A main effect of group was found (Supplementary material, Appendix 3, Table 6: parameter 6, -.2457 in cloglog hazard units, $p=.0159$, $\left.\mathrm{e}^{-.2457}=.78\right)$, indicating that the ASD children had an identification disadvantage in comparison to TD children: they had a systematically lower (cloglog) hazard than controls (see also Fig. 3). In other words, the hazard of event occurrence (i.e., correct identification) for the ASD group is estimated to be .78 times smaller than that for the TD group, in each frame number.

There were no significant interactions between participant group and any of the other predictors in our final model (see Appendix 3).

\section{Event History Analysis: Effects of Stimulus Characteristics (Across Both Participant Groups)}

There was a main effect of symmetry (parameter 12, 3526 in cloglog hazards, $p=.0004)$, which significantly changed over time (parameter 13-14), indicating that symmetrical objects had an identification advantage in earlier time frames (compared to non-symmetrical ones). This difference decreased in later time frames, and switched into an advantage for non-symmetrical objects (e.g., frame number 16: -.2815 in cloglog hazard units, $p=.0231$ ). An identification advantage for highly homogeneous (simple) objects (in comparison to complex objects) was found too (parameter 9: .5556 in cloglog hazard units, $p<.0001$ ).

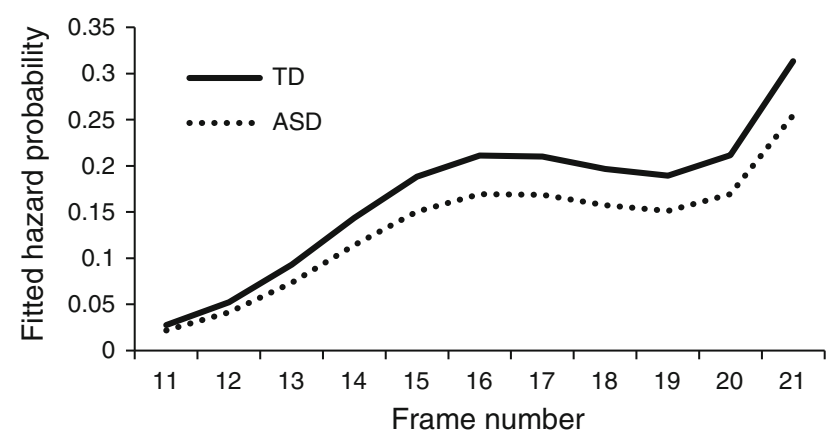

Fig. 4 A model-based estimated hazard plot, for complex (homogeneity $=0$ ), asymmetrical (symmetry $=0$ ) manmade (natural $=0$ ) objects, comparing the ASD group (dotted line) and the TD group (full line, i.e., baseline condition) in Experiment 1 (10-to-14-year olds)
The main effect of object category was not significant (parameter 7: .1332 in cloglog hazard units, $p=.1307$ ).

We identified several significant interactions between these stimulus characteristics: between object category and homogeneity (parameter 15), between object category and symmetry (parameters 16-17), and between homogeneity and symmetry (parameter 20). Since these did not interact with participant group and were in themselves not the focus of this study, they will not be discussed further. For more information, we refer to Appendix 3 and 4.

\section{Experiment 2: 6-to-10-Year Olds}

\section{Descriptive Statistics and Overall Results}

The TD group overall correctly identified outlines in $93.14 \%$ ( $\mathrm{SD}=.04)$ of the trials, which was significantly higher than the ASD group $(\mathrm{M}=89.28 \%, \mathrm{SD}=.05$; Mann-Whitney U-test: $\mathrm{z}=-2.64, p=.0084)$. There was no evidence for fewer or more attempts in providing an answer in the TD group (ASD group: $M=1.15$, $\mathrm{SD}=.08$; TD group: $\mathrm{M}=1.14$; $\mathrm{SD}=.13$; Mann-Whitney U-test: $\mathrm{z}=1.27, p=.2033$ ).

\section{Event History Analysis: General1 ${ }^{1}$}

The final (reduced) hazard model fitted at frame number 11 comprised 22 parameters, displayed in Appendix 5 (Supplementary material) together with their parameter estimates, standard errors, Chi square values, and $p$ values. In Appendix 6 (Supplementary material), the model based hazard plots of all conditions are displayed.

The hazard in the baseline condition (described by parameter 1-5, and displayed by the light-colored full line in Fig. 5), was low for the earlier frames, and increased to .14 around frame number 13 . In other words, the conditional probability that the average TD participant correctly identifies a baseline object (i.e., an asymmetrical, manmade object with low homogeneity) during frame number 13 equals .14 (given that the object has not been identified correctly before the start of frame 13). This conditional hazard probability decreased in the following frame numbers (hazard equals .11 for frame number 17), and then increased steeply (as in Experiment 1) and reached approximately $30 \%$ at the final frame number. The graph in Fig. 5 (dotted lines) also showed that the average ASD participant had a lower hazard probability

\footnotetext{
${ }^{1}$ Note. In Experiment 2, both male and female participants were included, whereas in Experiment 1, only boys participated. Fitting the reduced model at a subset of only male participants, yielded similar results.
} 
than the average TD participant, i.e., the average ASD participant had a lower conditional probability to correctly identify the object. However, due to the interaction between participant group (homogeneity) and time, the effect of participant group was not significant for all frame numbers. (This effect will be substantiated statistically in the next section.)

\section{Event History Analysis: Effect of Participant Group}

Although the ASD group had an identification disadvantage at all frame numbers (notice the negative sign in the parameter estimates, see Table 2), this disadvantage increased with time, and the main effect of participant group was only significant at later frames (e.g., from frame number 15 onwards). The interaction between participant group and homogeneity evolved significantly over time (parameter 18, see also Fig. 5). Graphical exploration of Fig. 5 and fitting the model at frames other than frame number 11 (see Table 2), indicated that the ASD group had an identification disadvantage for low homogeneous, thus complex objects (and not for simple ones). However, this interaction between participant group and homogeneity was only significant (parameter 16) at later frame numbers (e.g., frame number 19 onwards), and not in earlier ones (see, e.g., frame number 7-17, see Table 2). Graphical exploration of Fig. 5 also suggested that the ASD group had a slight disadvantage for highly homogeneous, simple objects (compare the dark full and dotted line) at intermediate time frames, but this difference was not significant in any of the frame numbers.

There were no significant interactions between participant groups and the other predictors (symmetry, object category) in our final model (see Appendix 6).

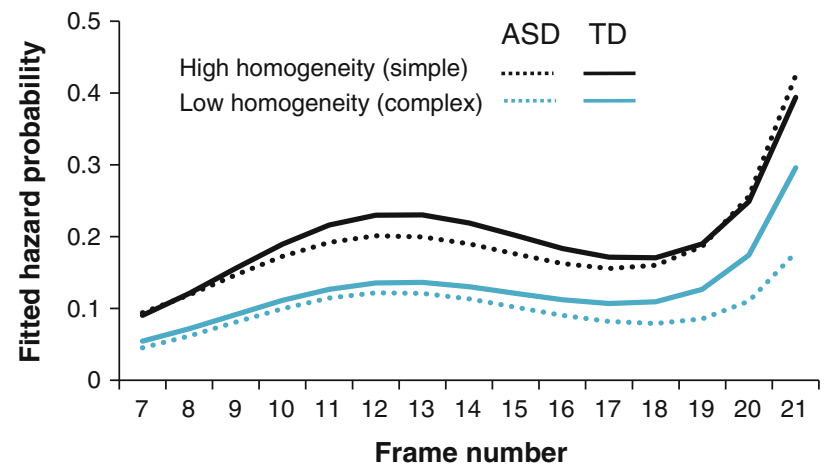

Fig. 5 The model-based estimated hazard plotting the evolution of the interaction effect between participant group and homogeneity over time, for the baseline objects (non-symmetrical, manmade), in Experiment 2 (6-to-10-year olds). The estimated hazard probability of the baseline is the light-colored full line (TD group, low homogeneity)
Event History Analysis: Effects of Stimulus Characteristics (Across Both Participant Groups)

There was a main effect of symmetry (parameter $9, .2713$ in cloglog hazard units, $p<.0001$ ), which significantly decreased linearly with discrete time (parameter 10), indicating that symmetrical objects had an identification advantage in earlier frame numbers, whereas this difference decreased in later frame numbers, and switched into an advantage for non-symmetrical objects at later time frames (e.g., frame number 15: parameter -.3891 in cloglog hazard units, $p=.0003$ ). The main effect of object category was significant (parameter 14, .1939 in cloglog hazard units, $p=.0049$ ), indicating an identification advantage for natural objects in comparison to manmade objects. This advantage of natural objects (in comparison to manmade ones) increased over time (parameter 15). An identification advantage was found for highly homogeneous, thus, simple objects in comparison to complex objects (parameter 11, .5848 in cloglog hazard units, $p<.0001$ ). This advantage decreased over time, and disappeared at the latest frames (e.g., frame number 21: parameter 11, .3550, $p=.2202$ ).

We identified several significant interactions between stimulus characteristics: between symmetry and object category (parameter 19-20), and between object category and homogeneity (parameters 21-22). Since these did not interact with participant group and were not in themselves the focus of this study, they will not be discussed further. We refer to the Supplementary material (Appendix 5 and 6) for more information.

\section{Discussion}

We investigated the atypical visual processing style in children with ASD, using an object identification task with Gaborized outlines of everyday objects. The outline and surface of each object gradually appeared in the stimulus sequence by aligning local Gabor elements from random orientation towards aligned. We applied our paradigm in two age groups of children with and without ASD. Although a direct statistical comparison of the results in both age groups (10-to-14 years old and 6-to-10 years old) is complicated by differences in the stimulus build-up, we found similar results in both age groups. However, the group difference seemed to be more subtle in the youngest sample (Experiment 2), in the sense that it emerged only later in the process (i.e., the identification disadvantage in the ASD group was only significant from frame number 15 onwards) and it interacted with stimulus complexity in that age sample.

Overall, children with ASD performed worse than TD children: they were less accurate, as indicated by the lower 
Table 2 Evolution of the parameter estimates and $p$ values ( $\operatorname{Pr}>$ Chi square) for the main effect of participant group and the interaction between participant group and homogeneity, for different time frames

\begin{tabular}{|c|c|c|c|c|c|c|c|c|}
\hline \multirow[t]{2}{*}{ Parameter } & \multicolumn{2}{|l|}{ Frame 7} & \multicolumn{2}{|l|}{ Frame 9} & \multicolumn{2}{|l|}{ Frame 11} & \multicolumn{2}{|l|}{ Frame 13} \\
\hline & Estimate & $p$ & Estimate & $p$ & Estimate & $p$ & Estimate & $p$ \\
\hline Group & -.1886 & .2668 & -.1299 & .2821 & -.1097 & .2701 & -.1278 & .1714 \\
\hline TB*group & & & & & .0005 & .9824 & & \\
\hline $\mathrm{TB}^{2} *$ group & & & & & -.0048 & .0952 & & \\
\hline Group*homogeneity & .2264 & .1501 & .0641 & .5038 & -.0225 & .7935 & -.0334 & .7259 \\
\hline TB*group*homogeneity & & & & & -.0244 & .3560 & & \\
\hline $\mathrm{TB}^{2 *}$ group*homogeneity & & & & & .0095 & .0370 & & \\
\hline \multirow[t]{2}{*}{ Parameter } & \multicolumn{2}{|l|}{ Frame 15} & \multicolumn{2}{|l|}{ Frame 17} & \multicolumn{2}{|l|}{ Frame 19} & \multicolumn{2}{|l|}{ Frame 21} \\
\hline & Estimate & $p$ & Estimate & $p$ & Estimate & $p$ & Estimate & $p$ \\
\hline $\begin{array}{l}\text { Group } \\
\mathrm{TB}^{*} \text { group } \\
\mathrm{TB}^{2 *} \text { group }\end{array}$ & -.1844 & .0430 & -.1844 & .0430 & -.4126 & .0005 & -.5843 & .0009 \\
\hline $\begin{array}{l}\text { Group*homogeneity } \\
\text { TB* group*homogeneity }^{*} \text { TB }^{2 * \text { group*homogeneity }}\end{array}$ & .0314 & .7741 & .0314 & .7741 & .3879 & .0867 & .6797 & .0522 \\
\hline
\end{tabular}

percentage correct identification (significant in Experiment 2 with the youngest age group, same trend but not significant in Experiment 1 with the oldest age group). Moreover, the event history analyses pointed out that the ASD group had an overall identification disadvantage, which remained pretty stable (Experiment 1) or increased (Experiment 2) over time: the hazard probability, and thus the chance to correctly identify an object (given that it was not identified before), was lower for the ASD group at every moment of the stimulus build-up. Moreover (see http://www.gestaltrevision.be/en/resources/supplementarymaterial for survivor plots), the average ASD participant correctly identified object contours at a later time frame than the average TD participant. We are confident that this effect of participant group is not merely a reflection of a slower motor response in the ASD group, since the ASD group also had a lower percentage of correct answers, independently of their response time (see sections "Descriptive Statistics and Overall Results"). The fact that the children with ASD had a lower correct identification at the final time frame too (a time frame in which there is no need to respond quickly), and the fact that this effect was modulated by one of the stimulus characteristics (complexity, in Experiment 2) strengthens us in our conviction that this group effect is not just caused by differences in reaction speed. Moreover, this main effect of participant group is also not due to any kind of hesitation to provide a-possibly incorrect-answer in the ASD group, since the children from the ASD group and the TD group provided an equal number of attempts per trial (see "Descriptive Statistics and Overall Results" sections).
Thus, the children with ASD need more information to identify an object outline (later responses), and, in addition, they perform worse at this task (lower accuracy). This reduced ability to identify the fragmented outlines might contradict their superior ability to detect predictable structures in stimuli (e.g., in the context of savantism: Mottron et al. 2013; and in the case of hyperlexia: Cobrinik 1982). Whereas superior pattern detection is often found in stimuli with a logical, inherent structure (Mottron et al. 2013), the nature of the organization is quite different in our study. In our task, the earliest relevant top-down matching predictions originate from one of the activated candidate object representations (based on the changing orientation signals). Only when a top-down prediction from a candidate representation matches the bottom-up input (to a certain extent), the outline will be correctly identified. Hence, successful "structure" identification in our paradigm requires the synergy of top-down and bottom-up processes, and any superior detection performance of predictable patterns will not help the children with ASD in our task. The similarities and differences between different kinds of "structures" and the enhanced or reduced performance in "structuring" in ASD requires further research.

Previous psychophysical and neurophysiological research in neurotypical adults already demonstrated the importance of stimulus characteristics on grouping and matching processes in the context of contour identification. However, not much was known about the importance of these characteristics in children. We demonstrate that midand high-level stimulus properties such as symmetry, 
object category (manmade versus natural objects), and visual complexity have a significant influence on the timecourse of object identification in children too. Since these main effects in themselves are not the most prominent focus of our study - we are rather interested in the similarities and differences between both participant groupswe will only briefly discuss these findings. First, consistent with multiple lines of research in adults, we show that symmetry has an early identification advantage. This finding is in correspondence with the known advantage of symmetrical objects during perceptual organization and it demonstrates that configural properties (such as symmetry) dominate early grouping processes (for a review, see Wagemans 1997). The decreasing influence of symmetry over time, as indicated by its interaction with time, suggests that the early grouping advantage for symmetrical objects decreases over time. Second, visually simple (highly homogeneous) object contours, which are known to have an a priori grouping advantage but a matching disadvantage, are more easily identified in comparison to complex contours (Donderi 2006; Panis and Wagemans 2009). Third, manmade objects have an identification disadvantage in comparison to natural objects in our sample (although this main effect was only significant in Experiment 2), which is in line with previous studies (Gerlach et al. 2004, 2006). However, these main effects are difficult to interpret on their own, since they are subtly modulated by other stimulus properties, as suggested by the interactions between different stimulus characteristics (see Appendix 3-6, Supplementary material). Note that the main effects of these variables were highly similar across both experiments (compare Appendix 3 and 5, Supplementary material). Readers interested in a more detailed discussion of how interactions between different stimulus characteristics can reflect the presence of so-called timecourse contingencies (indicative of feedback processing), can refer to Panis and Wagemans (2009).

More interestingly, we observed an interaction between participant group and homogeneity in the youngest age group (Experiment 2), indicating that children with ASD have a larger identification disadvantage for complex shapes, and this specific disadvantage increased over time (see also "Event History Analysis: Effect of Participant Group" section). Complex shapes are known to have an a priori early, bottom-up grouping disadvantage. However, once a complex shape outline is grouped, it activates fewer object presentations, resulting in a faster top-down matching. On the contrary, for simple shapes, which have an a priori grouping advantage and are easier to identify, the ASD group seemed to profit somewhat less in earlier time bins, although the group differences were not significant in these frames. This interaction effect strongly suggests that the demonstrated identification disadvantage in the ASD groups cannot be attributed to an early bottom-up grouping problem an sich. In that case, we would expect that the ASD group mainly had a disadvantage in the earlier time bins, when bottom-up grouping is mostly at play, and we also would have expected an interaction between group and symmetry (a factor which is known to have a strong bottom-up grouping advantage). Such a putative early, bottom-up grouping problem has also not been found in recent studies, as reviewed before (e.g., Del Viva et al. 2006; Kemner et al. 2007). Instead, this pattern of results emphasizes the importance of the interplay between different component processes in the later stages of the object identification process in children with ASD.

In the oldest age group (Experiment 1), though, we found an overall group difference throughout the complete time-course and we did not find evidence for a difference between children with and without ASD in the influence of the mid- and high-level stimulus characteristics during the identification process. Note that the lack of evidence for a more subtle group difference (fluctuating over time) in Experiment 1 can also be due to the more limited subset of relevant time frames in that experiment (virtually no children correctly identified the outline before frame 11 in Experiment 1). By accelerating the build-up in the initial time frames (when the stimulus changes do not give rise to major organizational changes yet) and slowing down the build-up afterwards, Experiment 2 yielded more interesting and more subtle effects over time. Unfortunately, we cannot disentangle the age difference and the differences in stimulus build-up between Experiments 1 and 2, so future research should evaluate that aspect in more detail. Moreover, additional experiments could examine the influence of low-level properties (such as fragment curvature and fragment length) on the time-course of object identification, and compare this between children with and without ASD.

Whereas classical visual processing models emphasized the strict (bottom-up) hierarchy of the visual system, recently developed theoretical accounts (Bar 2003; Bar et al. 2006) also underline the importance of recurrent (topdown) feedback mechanisms and horizontal connections. The hierarchical predictive coding model (Clark 2013; for an application in autism research, see Pellicano and Burr 2012; Van de Cruys et al. 2013), and the reverse hierarchy theory (Hochstein and Ahissar 2002), for instance, both emphasize the importance of the interplay between bottomup (feedforward) and top-down (feedback) mechanisms, and underline the differential contribution of these mechanisms in visual perception. Recent accounts in ASD also acknowledge the importance of attenuated top-down processing within and outside the visual processing stream (Liu et al. 2011; Loth et al. 2010; Mitchell et al. 2010), or enhanced low-level (bottom-up) processing (Samson et al. 
2012). In addition, and in line with the recently developed visual processing models, the results of our study support the role of top-down matching processes in object identification. The children with ASD might have more difficulties in considering alternative candidate object representations, or in top-down matching parts of the candidate representation to the visual input (e.g., correct spatial positions), or in flexibly switching between different alternative object hypotheses, or in iterating through the feedforward-andfeedback loops, and so forth. We clearly demonstrated a reduced accuracy, and a slower identification of outlines of everyday objects in ASD. However, we want to emphasize that the ASD group still performed pretty well: they correctly identified almost $90 \%$ of all cases. These results provide evidence for a suboptimal performance, and suggest that not one single process is completely affected in ASD. Instead, it seems that the interplay between stimulusdriven, bottom-up processes and top-down mechanisms is suboptimally adjusted in ASD.

Our paradigm entails a rich set of interacting processes, such as perceptual grouping along the contour and in the figure, segregation from the background, formation of a shape percept and an object representation, and matching to object descriptions and names in long-term memory. Moreover, several stimulus factors, some more local and others more global, could affect these processes and how they relate to one another during the execution of the main task of trying to make sense of the noisy and changing stimuli as soon as possible. Perhaps the complex and flexible interplay between component processes is also what is at stake in perceptual processing in everyday environments. We believe this could be a more promising avenue for future research of visual processing in ASD compared to the attempts to isolate specific subprocesses, which allow for simpler, more rigid and more controlled task execution, for which individuals with ASD can do similar or better (than TD participants). Although simpler paradigms targeting a single process (believed to be representative for "local" or "global" processing) or a single stimulus factor (relevant for low-, mid- or high-level processes) have the advantage of more experimental control, they also do afford learning of optimal strategies and more rigid execution of strategies, while they are less representative for the complexity of processing in everyday life.

Acknowledgments We are grateful to all participating children and their parents. Special thanks to the participating special needs school, Ten Bunderen (Moorslede), and two mainstream schools, De Puzzel (Kleine-Brogel) and De Schommel (Lommel). Thanks to Bart Machilsen for stimulus construction (Experiment 2). We thank Birgitt Haesen and the following master students for their assistance with data collection: Nele Berghmans, Stéphanie Deckmyn, Sanne Drees, Tine Herreman, Ellen Janssen, Loes Steegmans, Lotte van Esch, Alysée Van Laeken, and Leen Vercammen. This research was funded by the Methusalem program by the Flemish Government (METH 08/
02) awarded to Johan Wagemans and a grant from the Research Council of the KU Leuven (IDO/080/013) awarded to Jean Steyaert, Johan Wagemans, and Ilse Noens.

\section{References}

Allison, P. D. (2010). Survival analysis using SAS: A practical guide. Second Edition: SAS Institute.

American Psychiatric Association. (2000). Diagnostic and statistical manual of mental disorders (4th edition text revision). Washington, DC: American Psychiatric Association.

Bar, M. (2003). A cortical mechanism for triggering top-down facilitation in visual object recognition. Journal of Cognitive Neuroscience, 15(4), 600-609. doi:10.1162/089892903321662976.

Bar, M., Kassam, K. S., Ghuman, A. S., Boshyan, J., Schmidt, A. M., Dale, A. M., et al. (2006). Top-down facilitation of visual recognition. Proceedings of the National Academy of Sciences of the United States of America, 103(2), 449-454. doi:10.1073/ pnas.0507062103.

Barttfeld, P., Wicker, B., Cukier, S., Navarta, S., Lew, S., \& Sigman, M. (2011). A big-world network in ASD: Dynamical connectivity analysis reflects a deficit in long-range connections and an excess of short-range connections. Neuropsychologia, 49(2), 254-263. doi:10.1016/j.neuropsychologia.2010.11.024.

Behrmann, M., Thomas, C., \& Humphreys, K. (2006). Seeing it differently: Visual processing in autism. Trends in Cognitive Sciences, 10(6), 258-264. doi:10.1016/j.tics.2006.05.001.

Blake, R., Turner, L. M., Smoski, M. J., Pozdol, S. L., \& Stone, W. L. (2003). Visual recognition of biological motion is impaired in children with autism. Psychological Science, 14(2), 151-157. doi:10.1111/1467-9280.01434.

Clark, A. (2013). Whatever next? Predictive brains, situated agents, and the future of cognitive science. Behavioral and Brain Sciences, 36(3), 1-73.

Cobrinik, L. (1982). The performance of hyperlexic children on an incomplete words task. Neuropsychologia, 20(5), 569-577. doi:10.1016/0028-3932(82)90030-6.

Constantino, J. N. (2002). The social responsiveness scale. Los Angelos: Western Psychological Services.

Dakin, S., \& Frith, U. (2005). Vagaries of visual perception in autism. Neuron, 48, 497-507. doi:10.1016/j.neuron.2005.10.018.

Dale, G., \& Arnell, K. M. (2013). Investigating the stability of and relationships among global/local processing measures. Attention, perception \& psychophysics, 75(3), 394-406. doi:10.3758/ s13414-012-0416-7.

Del Viva, M. M., Igliozzi, R., Tancredi, R., \& Brizzolara, D. (2006). Spatial and motion integration in children with autism. Vision Research, 46(8-9), 1242-1252. doi:10.1016/j.visres.2005.10.018.

Donderi, D. C. (2006). Visual complexity: A review. Psychological Bulletin, 132(1), 73-97. doi:10.1037/0033-2909.132.1.73.

Evers, K., Noens, I., Steyaert, J., \& Wagemans, J. (2011). Combining strengths and weaknesses in visual perception of children with an autism spectrum disorder: Perceptual matching of facial expressions. Research in Autism Spectrum Disorders, 5(4), 1327-1342. doi:10.1016/j.rasd.2011.01.004.

Falter, C. M., \& Bailey, A. J. (2012). Perception of mirror symmetry in autism spectrum disorders. Autism, 16(6), 622-626. doi:10. $1177 / 1362361311407353$.

Frith, U., \& Happé, F. (1994). Autism-beyond theory of mind. Cognition, 50(1-3), 115-132. doi:10.1016/0010-0277(94)90024-8.

Gerlach, C., Law, I., \& Paulson, O. B. (2004). Structural similarity and category-specificity: A refined account. Neuropsychologia, 42(11), 1543-1553. doi:10.1016/j.neuropsychologia.2004.03.004. 
Gerlach, C., Law, I., \& Paulson, O. B. (2006). Shape configuration and category-specificity. Neuropsychologia, 44(7), 1247-1260. doi:10.1016/j.neuropsychologia.2005.09.010.

Gotham, K., Pickles, A., \& Lord, C. (2009). Standardizing ADOS scores for a measure of severity in autism spectrum disorders. Journal of Autism and Developmental Disorders, 39(5), 693705. doi:10.1007/s10803-008-0674-3.

Gotham, K., Risi, S., Dawson, G., Tager-Flusberg, H., Joseph, R., Carter, A., et al. (2008). A replication of the Autism Diagnostic Observation Schedule (ADOS) revised algorithms. Journal of the American Academy of Child and Adolescent Psychiatry, 47(6), 642-651. doi:10.1097/Chi.0b013e31816bffb7.

Happé, F., \& Booth, R. D. L. (2008). The power of the positive: Revisiting weak coherence in autism spectrum disorders. The Quarterly Journal of Experimental Psychology, 61(1), 50-63. doi:10.1080/17470210701508731.

Happé, F., \& Frith, U. (2006). The weak coherence account: Detailfocused cognitive style in autism spectrum disorders. Journal of Autism and Developmental Disorders, 36(1), 5-25. doi:10.1007/ s10803-005-0039-0.

Hess, R., \& Field, D. (1999). Integration of contours: New insights. Trends in Cognitive Sciences, 3(12), 480-486. doi:10.1016/ S1364-6613(99)01410-2.

Hess, R., Hayes, A., \& Field, D. (2003). Contour integration and cortical processing. Journal of Physiology-Paris, 97(2-3), 105119. doi:10.1016/j.jphysparis.2003.09.013.

Hochstein, S., \& Ahissar, M. (2002). View from the top: Hierarchies and reverse hierarchies in the visual system. Neuron, 36(5), 791804. doi:10.1016/S0896-6273(02)01091-7.

Humphreys, G., Riddoch, M., \& Quinlan, P. (1988). Cascade processes in picture identification. Cognitive Neuropsychology, 5(1), 67-103. doi:10.1080/02643298808252927.

Kanner, L. (1943). Autistic disturbances of affective contact. Nervous Child, 2, 217-250.

Kemner, C., Lamme, V. A. F., Kovacs, I., \& van Engeland, H. (2007). Integrity of lateral and feedbackward connections in visual processing in children with pervasive developmental disorder. Neuropsychologia, 45, 1293-1298. doi:10.1016/j.neuropsychologia.2006.09.016.

Liu, Y., Cherkassky, V. L., Minshew, N. J., \& Just, M. A. (2011). Autonomy of lower-level perception from global processing in autism: Evidence from brain activation and functional connectivity. Neuropsychologia, 49(7), 2105-2111. doi:10.1016/j. neuropsychologia.2011.04.005.

Lord, C., Risi, S., Lambrecht, L., Cook, E. H., Leventhal, B. L., DiLavore, P. C., et al. (2000). The Autism Diagnostic Observation Schedule-Generic: A standard measure of social and communication deficits associated with the spectrum of autism. Journal of Autism and Developmental Disorders, 30(3), 205223. doi:10.1023/A:1005592401947.

Loth, E., Gomez, J. C., \& Happé, F. (2010). When seeing depends on knowing: Adults with autism spectrum conditions show diminished top-down processes in the visual perception of degraded faces but not degraded objects. Neuropsychologia, 48(5), 12271236. doi:10.1016/j.neuropsychologia.2009.12.023.

Milne, E., \& Szczerbinski, M. (2009). Global and local perceptual style, field-independence, and central coherence: An attempt at concept validation. Advances in Cognitive Psychology, 5, 1-26. doi:10.2478/v10053-008-0062-8.

Mitchell, P., Mottron, L., Soulieres, I., \& Ropar, D. (2010). Susceptibility to the Shepard illusion in participants with autism: Reduced top-down influences within perception? Autism Research, 3(3), 113-119. doi:10.1002/aur.130.

Mottron, L., Bouvet, L., Bonnel, A., Samson, F., Burack, J. A., Dawson, M., et al. (2013). Veridical mapping in the development of exceptional autistic abilities. Neuroscience and Biobehavioral Reviews, 37(2), 209-228. doi:10.1016/j.neubiorev.2012.11.016.
Mottron, L., \& Burack, J. A. (2001). Enhanced perceptual functioning in the development of autism. In J. A. Burack, T. Charman, N. Yirmiya, \& P. R. Zelazo (Eds.), The development of autism: Perspectives from theory and research (pp. 131-148). Mahwah, NJ: Erlbaum.

Mottron, L., Dawson, M., Soulières, I., Hubert, B., \& Burack, J. (2006). Enhanced perceptual functioning in autism: An update, and eight principles of autistic perception. Journal of Autism and Developmental Disorders, 36(1), 27-43. doi:10.1007/s10803005-0040-7.

Panis, S., \& Hermens, F. (2013). Time course of spatial contextual interference: Event history analyses of simultaneous masking by nonoverlapping patterns. Journal of Experimental Psychology: Human Perception and Performance. doi:10.1037/a0032949.

Panis, S., \& Wagemans, J. (2009). Time-course contingencies in perceptual organization and identification of fragmented object outlines. Journal of Experimental Psychology-Human Perception and Performance, 35(3), 661-687. doi:10.1037/A0013547.

Pellicano, E., \& Burr, D. (2012). When the world becomes "too real": A Bayesian explanation of autistic perception. Trends in Cognitive Sciences,. doi:10.1016/j.tics.2012.08.009.

Perreault, A., Gurnsey, R., Dawson, M., Mottron, L., \& Bertone, A. (2011). Increased sensitivity to mirror symmetry in autism. PLoS ONE, 6(4), e19519. doi:10.1371/journal.pone.0019519.

Roeyers, H., Thys, M., Druart, C., De Schryver, M., \& Schittekatte, M. (2012). SRS: Screeningslijst voor autismespectrum stoornissen. Amsterdam: Hogrefe.

Ronald, A., \& Happé, F. (2008). The "fractionable autism triad": A review of evidence from behavioural, genetic, cognitive and neural research. Neuropsychology Review, 18(4), 287-304. doi:10.1007/s11065-008-9076-8.

Samson, F., Mottron, L., Soulieres, I., \& Zeffiro, T. A. (2012). Enhanced visual functioning in autism: An ALE meta-analysis. Human Brain Mapping, 33(7), 1553-1581. doi:10.1002/hbm.21307.

Sassi, M., Vancleef, K., Machilsen, B., Panis, S., \& Wagemans, J. (2010). Identification of everyday objects on the basis of Gaborized outline versions. Perception, 39, 184.

Sattler, J. M. (2001). Assessment of children: Cognitive applications (4th ed.). San Diego, CA: Jerome M Sattler Publisher Inc.

Shah, A., \& Frith, U. (1983). An islet of ability in autistic children: A research note. Journal of Child Psychology and Psychiatry and Allied Disciplines, 24, 613-620. doi:10.1111/j.1469-7610.1983. tb00137.x.

Simmons, D. R., Robertson, A. E., McKay, L. S., Toal, E., McAleer, P., \& Pollick, F. E. (2009). Vision in autism spectrum disorders. Vision Research, 49(22), 2705-2739. doi:10.1016/j.visres.2009. 08.005 .

Singer, J. D., \& Willett, J. B. (2003). Applied longitudinal data analysis: Modeling change and event occurence. New York: Oxford University Press.

Snodgrass, J., \& Vanderwart, M. (1980). Standardized set of 260 pictures-Norms for name agreement, image agreement, familiarity, and visual complexity. Journal of Experimental Psychology-Human Learning and Memory, 6(2), 174-215. doi:10.1037/ 0278-7393.6.2.174.

Torfs, K., Panis, S., \& Wagemans, J. (2010). Identification of fragmented object outlines: A dynamic interplay between different component processes. Visual Cognition, 18(8), 11331164. doi:10.1080/13506281003693593.

Van de Cruys, S., de-Wit, L., Evers, K., Boets, B., \& Wagemans, J. (2013). Weak priors versus overfitting of predictions in autism: Reply to Pellicano and Burr (TICS, 2012). i-Comment, 4, 95-97. doi:10.1068/i0580ic.

Wagemans, J. (1997). Characteristics and models of human symmetry detection. Trends in Cognitive Sciences, 1(9), 346-352. doi:10. 1016/S1364-6613(97)01105-4. 
Wagemans, J., De Winter, J., Op de Beeck, H. P., Ploeger, A., Beckers, T., \& Vanroose, P. (2008). Identification of everyday objects on the basis of silhouette and outline versions. Perception, 37(2), 207-244. doi:10.1068/p5825.

Wagemans, J., Elder, J. H., Kubovy, M., Palmer, S. E., Peterson, M. A., Singh, M., et al. (2012). A century of Gestalt psychology in visual perception: I. Perceptual grouping and figure-ground organization. Psychological Bulletin, 138(6), 1172-1217. doi:10. 1037/a0029333.
Wass, S. (2011). Distortions and disconnections: Disrupted brain connectivity in autism. Brain and Cognition, 75(1), 18-28. doi:10.1016/j.bandc.2010.10.005.

Wechsler, D. (1992). Wechsler intelligence scale for children (3rd ed.). London: The Psychological Corporation.

Wing, L., Gould, J., \& Gillberg, C. (2011). Autism spectrum disorders in the DSM-V: Better or worse than the DSM-IV? Research in Developmental Disabilities, 32(2), 768-773. doi:10.1016/j.ridd. 2010.11.003. 